\title{
Recurrent Body Rash Warranted Second Desensitization With Acyclovir in a Myeloma Patient: A Case Report
}

\author{
Jack T. Seki ${ }^{a}$ c, d , Pamela Nga, Wallace Lam ${ }^{a}$, Julie Cote ${ }^{\mathrm{b}}$, \\ Anca Prica ${ }^{b}$
}

\begin{abstract}
A 75-year-old woman developed a moderately severe rash about a week and a half after the start of bortezomib (Btb)-based chemotherapy for IgG lambda multiple myeloma; at the time, she was also receiving acyclovir as antiviral prophylaxis in addition to herpes zoster $(\mathrm{HZ})$ vaccination. $\mathrm{HZ}$ reactivation rate is high in Btb recipients; therefore, the timing of antiviral prevention is critical in relation to Btb. Attempts were made to identify the offending agent based on the timing of drugs administered and the appearance of skin lesions in relation to other drugs. Both Btb and acyclovir were potential culprits. However, the timing of rash presented on days $9-10$ revealed the offending agent when the corticosteroid was weaned off while acyclovir continued. A decision was made to administer acyclovir rapid desensitization program (RDP) for our patient.
\end{abstract}

Keywords: Hypersensitivity; Antiviral agent; Cutaneous reactions

\section{Introduction}

In a myeloma practice guideline for patients receiving bortezomib (Btb), antiviral prophylaxis should be considered in the prevention of herpes zoster $(\mathrm{HZ})$ reactivation [1]. This was supported by the reduced viability of the T lymphocytes to proliferate when exposed to Btb [2]. The evidence is even more compelling in terms of reporting higher incidences of $\mathrm{HZ}$ when these patients with impaired cellular immunity received Btb in combination with dexamethasone than those taking dexamethasone as a single agent [3]. When the active prevention

Manuscript submitted September 28, 2016, accepted October 6, 2016

aDepartment of Pharmacy, Princess Margaret Cancer Centre, University Health Network, Toronto, Ontario M5G 2M9, Canada

bepartment of Medical Oncology and Hematology, Princess Margaret Cancer Centre, University Health Network, Toronto, Ontario M5G 2M9, Canada 'Leslie Dan Faculty of Pharmacy, University of Toronto, Toronto, Ontario M5S 3M2, Canada

${ }^{\mathrm{d} C}$ Corresponding Author: Jack T. Seki, Department of Pharmacy, Princess Margaret Cancer Centre, University Health Network, 610 University Ave., Toronto, Ontario M5G 2M9, Canada. Email: Jack.seki@uhn.ca

doi: https://doi.org/10.14740/jocmr2772w duration is prolonged over a median of 16 weeks, the incidence of $\mathrm{HZ}$ recurrence has been shown to decrease significantly [4]. The same was demonstrated in those receiving acyclovir prophylaxis up to 4 weeks after the last dose of chemotherapy [5]. When patients encountered infrequent hypersensitivity reactions to acyclovir, a selection of other alternative antiviral agents may be considered. After thorough assessment of our patient and weighing safety vs. toxicity to the potential antiviral list, it was clear that acyclovir remained the sound drug of choice. Given the limited experience, desensitization was the best means to safely re-introduce acyclovir back to our patient. To the best of our knowledge, we described the first tandem rapid desensitization program (RDP) performed to a myeloma patient receiving Btb-based therapy.

\section{Case Report}

We report a 75-year-old woman who looks younger than stated age, with a standing history of allergic reactions to penicillin and ibuprofen from which she developed a rash and angioedema, respectively. She was diagnosed with $\mathrm{ER}+, \mathrm{PR}+$ and HER2+ localized advanced breast cancer in 2008, and was successfully treated. She was well until the fall of 2014 when she presented with lytic bony metastases to her L2, L5, right acetabulum and right pelvic bone with cortical destruction. Over the course of the following year, her renal function gradually deteriorated while receiving radiation therapy to her bony lesions. Renal biopsy revealed light chain lambda cast nephropathy. Complete workup included a bone marrow aspirate and biopsy which confirmed the diagnosis of multiple myeloma. She initiated the first cycle of chemotherapy consisting of Btb (Velcade ${ }^{\mathbb{R}}$ ) $1.3 \mathrm{mg} / \mathrm{m}^{2}$ subcutaneously (SC) on days 1 , 8,15 and 22, melphalan $9 \mathrm{mg} / \mathrm{m}^{2}$ orally on days $1-4$ and prednisone $100 \mathrm{mg}$ orally on days $1-4$, with a subsequent 5 -day taper. The cycle was repeated every 35 days. By this time, she had had day 1 and day 8 doses of Btb. Acyclovir orally $400 \mathrm{mg}$ bid was concurrently given for shingles prophylaxis. The patient had also received a dose of $\mathrm{HZ}$ vaccine for added protection (unaware to the oncologist). About days 9 - 10 of starting this regimen, she developed a very extensive purpuric itchy rash (Figs. 1 and 2 ) involving $>50 \%$ of her body which began around her shin and spread quickly to her upper legs, abdomen, upper torso and shoulders. While the day $15 \mathrm{Btb}$ was held, her rash worsened. On day 16, acyclovir was also put 


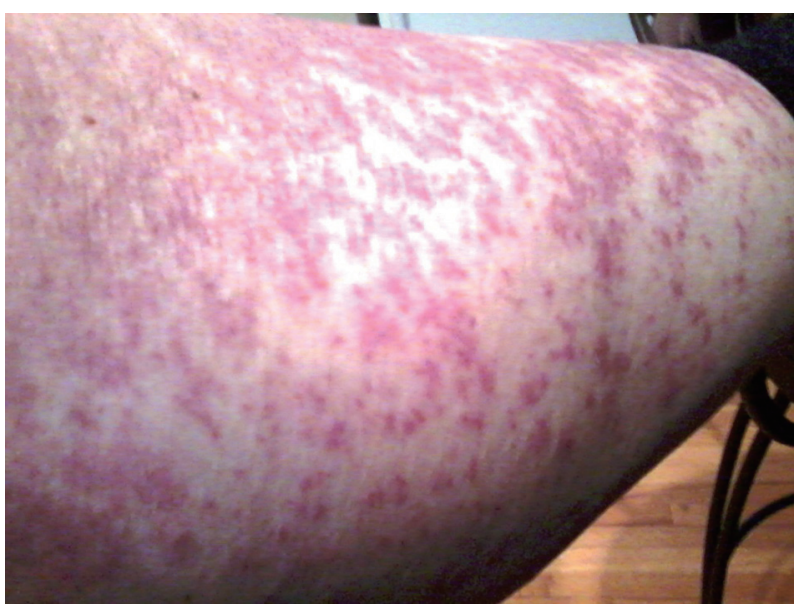

Figure 1. Erythematous, maculopapular rash on upper thigh.

on hold, and the rash began showing signs of improvement within the next few days. The rash was described as confluent maculopapular, erythematous, blanchable, slightly raised, and limited to the described areas. This was not associated with petechial lesions. Her platelets were within normal limits. She did not have fever, but the rash was pruritic. Other than her elevated serum creatinine $(177 \mu \mathrm{mol} / \mathrm{L})$, she was borderline anemic, normotensive, and afebrile and liver function tests were within normal limits. The skin lesions were highly suggestive of an adverse drug reaction. Pharmacy was consulted for probable acyclovir-induced rash and the decision to initiate RDP was made. About $4 \mathrm{~h}$ after completion of the acyclovir RDP on February 17, 2016, patient reported dryness and pruritus around her upper arms/shoulders areas. Moisturizing cream was applied without any effect. Diphenhydramine 50 mg orally $\times 1$ was administered without resolution. By that time, her pruritic, erythematous rash with even distribution appeared over her shin and was spreading upward toward her abdomen. A dose of methylprednisolone was given and the rash subsided quickly within a few hours. She was given a second desensitization on February 25, 2016 a week after the initial desensitization, and a rash re-appeared again about $4 \mathrm{~h}$ after second RDP, but it was much less dramatic and resolved on its own without any intervention overnight. Patient was re-challenged with acyclovir $400 \mathrm{mg}$ in the morning without incident, and discharged a few hours later. She remained on $400 \mathrm{mg}$ twice a day without any side effects. The patient was not taking penicillin or ibuprofen before the occurrence of rash.

\section{Discussion}

We believe this was the first acyclovir RDP that was conducted successfully in two consecutive attempts in a multiple myeloma patient. Although Btb was thought to be the likely culprit for the rash, acyclovir was also probable, given the proximity of the drug administration times. To determine causality, we looked at the incidence of skin adverse reactions to both Btb and acyclovir.

Adverse reactions reported on skin and SC tissue disor-

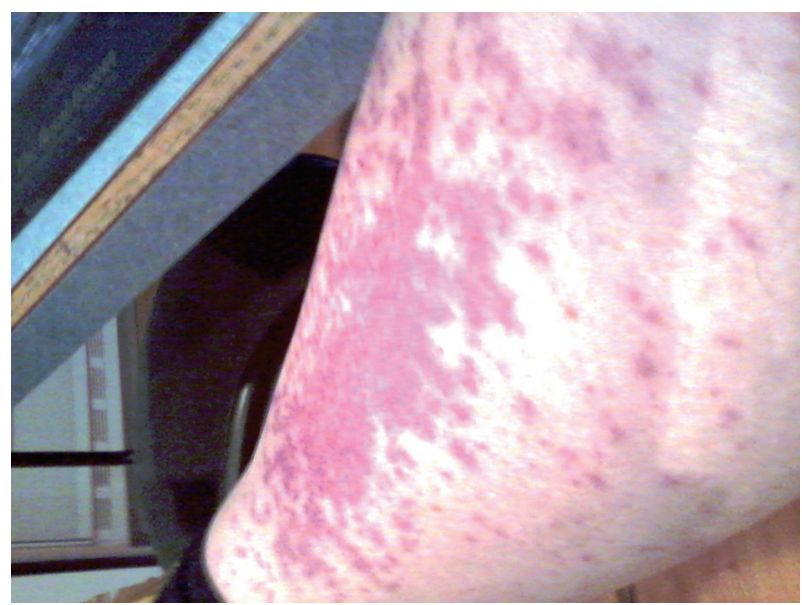

Figure 2. Erythematous, maculopapular rash on lower leg.

ders (all grades, and grade $\geq 3$ ) associated with SC vs. intravenous (IV) Btb were 35 (24\%), and 4 (3\%), vs. $13(18 \%)$, and 0 , respectively [6]. Other case reports have typically described cutaneous toxicity as localized reactions at the SC injection sites $[7,8]$. In one report from 11 of 28 patients $(39 \%)$, the SC site reactions were described as redness $(5,18 \%)$, rash $(3,11 \%)$, pain/tenderness $(3,11 \%)$, itching $(2,7 \%)$, and burning $(1,4 \%)$ [9]. Some of the skin eruptions occurred at each cycle of drug administration, and dissipated after treatment without systemic involvement, with lesions typically described as lymphocytic infiltration preventable with corticosteroids [10]. Skin toxicity or injection site reactions of $\geq$ grade 2 occurred more frequently in patients on the first cycle than the second cycle of Btb, and in a case subsided quickly with a dose of prednisone, and recurrent reaction was preventable with pre-dose steroid $[11,12]$. Punch biopsy results describe these rashes as vasculitic in nature due to the release of pro-inflammatory cytokine, which is thought to be through the inhibition of the NF-kB versus an immunologically linked mechanism $[7,13]$. The underlying mechanism has been proposed as an inflammatory response that may be caused by 1) delayed hypersensitivity, 2) cell-mediated immune responses, 3 ) vascular damage, and 4) direct toxic reaction by the proteasome inhibitor [14]. Similarly, in a comparative study of IV vs. SC Btb where patients received pre-dose steroids, no reaction was reported in the IV arm, whereas low grade localized, and self-limiting injection reactions were reported in the SC arm $[3,15]$. Although redness is commonly reported (84/147, $57 \%$ ) [6], Btb SC seemed to be better tolerated than IV and associated with fewer overall adverse reactions [3, 16, 17$]$. Topical steroid can be a useful local remedy (up to grade 2 CTCAE 4) [11].

Our patient was receiving prednisone as part of the VMP (SC Velcade ${ }^{\circledR}$ was given) regimen, and no apparent adverse reaction was reported during chemotherapy. A few days after Btb, during the steroid tapering, a localized mild redness was visible at the injection site around the abdomen from the first dose, of first cycle Btb injection. This was consistent with the aforementioned reports. Since the generalized maculopapular rash erupted at the end of the steroid cycle, the immediate re- 
sponse would be to hold further day 15 Btb dosing. However, the index of suspicion grew stronger towards acyclovir, because the patient was still on acyclovir at that time. When the rash gradually resolving days after the acyclovir was being put on hold, we felt quite confident that the skin lesion was most likely due to acyclovir. This may be explained by the fact that no visible rash occurred in our patient when prednisone was still in the system during the first 4 days of Btb, and the following few days of the steroid tapering effect. The rash only became apparent soon after at the end of steroid tapering. We made the conscious decision then to perform acyclovir desensitization not only because the patient needed continuous antiviral prophylaxis while receiving proteasome inhibitor treatments $[1,2,4,5,12,18-20]$, but also having constant unnecessary steroid exposure was contraindicated.

Acyclovir-associated hypersensitivity has been rarely described in immunocompromised patients, and to address the adverse reactions, RDP has been used with varying degrees of success [21-23]. Although a repeat desensitization may be warranted enabling patients to continue treatment, experience has been scarcely documented in myeloma.

Cancer patients such as ours who developed hypersensitivity reactions to acyclovir have limited alternatives. Valacyclovir, ganciclovir and famciclovir have 2-aminopurine as their core chemical structure, making cross-sensitivity likely [24]. Valacyclovir is the L-valyl ester of acyclovir, and when metabolized via hydroxylase enzyme system in the gut, is converted to high concentration of acyclovir [25]. Famciclovir as a prodrug is actively and efficiently biotransformed via double deacetylation to penciclovir a potent anti-HSV in the gut, liver and blood [26]. Bayrou et al [27] described a case where a patient had shown negative patch test after taking famciclovir but the results of the provocation test elicited a pruritic rash 12 $\mathrm{h}$ after ingestion for the first time. Based on this, famciclovir is also not likely a feasible option for our patient. Penciclovir is topical and not useful. Cidofovir has serious renal toxicity which is common and administered intravenously, therefore a poor choice for our ambulatory patient [28]. In addition to elevation of serum creatinine, ganciclovir-related reversible immunosuppression is well known, but is unsuitable for our patients undergoing maintenance chemotherapy [29]. While foscarnet may be another option, its renal toxicity and electrolyte disturbance potential are quite common, making it a none ideal candidate for our patient with deteriorating myeloma kidneys [29]. Given limited choices, we resort to acyclovir as an $\mathrm{HZ}$ prevention measure. The doses used varied from center to center $[4,20,30]$.

\section{Conclusion}

Although a full blown body rash by end of steroid tapering solicited a high degree of suspicion that acyclovir was the cause, the recurrence of rash after completion of the initial RDP confirmed that acyclovir was the inciting agent. We felt that it was appropriate to conduct RDP due to the lack of better alternatives for $\mathrm{HZ}$ prophylaxis. Our decision to re-administer the second RDP was made based on the recurrent rash that required medical intervention. Would the patient have done well upon a re-challenge with a full dose acyclovir without a repeated RDP? While the recurrence of rash after the second RDP was worrisome, it was mild and self-limiting. It was gratifying to see that our patient continued to be rash-free while being maintained on acyclovir $400 \mathrm{mg}$ bid for $\mathrm{HZ}$ prophylaxis since RDP began in February 25, 2016.

\section{Conflicts of Interest}

Jack T. Seki received honoraria from Astellas, Merck, Pfizer, Leo, and study grant from Astellas, Merck, Sanofi, and Amgen. Pamela Ng: none. Wallace Lam: none. Julie Cote: honoraria: Takeda and Janssen. Anca Prica: none.

\section{References}

1. Kouroukis CT, Baldassarre FG, Haynes AE, Imrie K, Reece DE, Cheung MC. Bortezomib in multiple myeloma: a practice guideline. Clin Oncol (R Coll Radiol). 2014;26(2):110-119.

2. Blanco B, Perez-Simon JA, Sanchez-Abarca LI, Carvajal-Vergara X, Mateos J, Vidriales B, Lopez-Holgado N, et al. Bortezomib induces selective depletion of alloreactive T lymphocytes and decreases the production of Th1 cytokines. Blood. 2006;107(9):3575-3583.

3. Richardson PG, Sonneveld P, Schuster MW, Irwin D, Stadtmauer EA, Facon T, Harousseau JL, et al. Bortezomib or high-dose dexamethasone for relapsed multiple myeloma. N Engl J Med. 2005;352(24):2487-2498.

4. Vickrey E, Allen S, Mehta J, Singhal S. Acyclovir to prevent reactivation of varicella zoster virus (herpes zoster) in multiple myeloma patients receiving bortezomib therapy. Cancer. 2009;115(1):229-232.

5. Swaika A, Paulus A, Miller KC, Sher T, Almyroudis NG, Ball D, Wood M, et al. Acyclovir prophylaxis against varicella zoster virus reactivation in multiple myeloma patients treated with bortezomib-based therapies: a retrospective analysis of 100 patients. J Support Oncol. 2012;10(4):155-159.

6. Moreau P, Pylypenko H, Grosicki S, Karamanesht I, Leleu X, Grishunina M, Rekhtman G, et al. Subcutaneous versus intravenous administration of bortezomib in patients with relapsed multiple myeloma: a randomised, phase 3, non-inferiority study. Lancet Oncol. 2011;12(5):431-440.

7. Sanchez-Politta S, Favet L, Kerl K, Dietrich PY, Piguet V. Bortezomib-induced skin eruption. Dermatology. 2008;216(2):156-158.

8. Obeid KM, Ferrara R, Sharma M. Cutaneous lesion induced by a subcutaneous administration of bortezomib. Clin Lymphoma Myeloma Leuk. 2012;12(4):284-286.

9. Barbee MS, Harvey RD, Lonial S, Kaufman JL, Wilson NM, McKibbin T, Hutcherson DA, et al. Subcutaneous versus intravenous bortezomib: efficiency practice variables and patient preferences. Ann Pharmacother. 2013;47(9):1136-1142.

10. Agterof MJ, Biesma DH. Images in clinical Medicine. Bortezomib-induced skin lesions. N Engl J Med. 
2005;352(24):2534.

11. Kamimura T, Miyamoto T, Yokota N, Aoki T, Ito Y, Akashi K. High incidence and severity of injection site reactions in the first cycle compared with subsequent cycles of subcutaneous bortezomib. Int J Hematol. 2013;98(6):694701.

12. Pour L, Hajek R, Zdenek A, Krejci M, Krivanova A, Vorlicek J. Skin lesions induced by bortezomib. Haematologica. 2005;90(12 Suppl):ECR44.

13. Gerecitano J, Goy A, Wright J, MacGregor-Cortelli B, Neylon E, Gonen M, Esseltine D, et al. Drug-induced cutaneous vasculitis in patients with non-Hodgkin lymphoma treated with the novel proteasome inhibitor bortezomib: a possible surrogate marker of response? $\mathrm{Br} \mathrm{J}$ Haematol. 2006;134(4):391-398.

14. Wu KL, Heule F, Lam K, Sonneveld P. Pleomorphic presentation of cutaneous lesions associated with the proteasome inhibitor bortezomib in patients with multiple myeloma. J Am Acad Dermatol. 2006;55(5):897-900.

15. Lamm W, Drach-Schauer B, Eder S, Drach J. Bortezomib administered subcutaneously is well tolerated in bortezomib-based combination regimens used in patients with multiple myeloma. Oncology. 2013;85(4):223-227.

16. Ng P, Incekol D, Lee R, Paisley E, Dara C, Brandle I, Kaufman M, et al. Tolerability of Velcade (Bortezomib) subcutaneous administration using a maximum volume of $3 \mathrm{~mL}$ per injection site. J Oncol Pharm Pract. 2015;21(4):285-292.

17. Reyes-Habito CM, Roh EK. Cutaneous reactions to chemotherapeutic drugs and targeted therapies for cancer: part I. Conventional chemotherapeutic drugs. J Am Acad Dermatol. 2014;71(2):203 e201-203 e212; quiz 215-206.

18. Chanan-Khan A, Sonneveld P, Schuster MW, Stadtmauer EA, Facon T, Harousseau JL, Ben-Yehuda D, et al. Analysis of herpes zoster events among bortezomibtreated patients in the phase III APEX study. J Clin Oncol. 2008;26(29):4784-4790.

19. Mateos MV, Hernandez JM, Hernandez MT, Gutierrez NC, Palomera L, Fuertes M, Diaz-Mediavilla J, et al. Bortezomib plus melphalan and prednisone in elderly un- treated patients with multiple myeloma: results of a multicenter phase 1/2 study. Blood. 2006;108(7):2165-2172.

20. Aoki T, Nishiyama T, Imahashi N, Kitamura K. Efficacy of continuous, daily, oral, ultra-low-dose $200 \mathrm{mg}$ acyclovir to prevent herpes zoster events among bortezomibtreated patients: a report from retrospective study. Jpn J Clin Oncol. 2011;41(7):876-881.

21. Snape SE, Finch RG, Venkatesan P. Aciclovir desensitisation and rechallenge. BMJ Case Rep. 2011;2011.

22. Henry RE, Wegmann JA, Hartle JE, Christopher GW. Successful oral acyclovir desensitization. Ann Allergy. 1993;70(5):386-388.

23. Kawsar M, Parkin JM, Forster G. Graded challenge in an aciclovir allergic patient. Sex Transm Infect. 2001;77(3):204-205.

24. Schuster J, Fabri M, Eming S, Hunzelmann N. Allergic drug eruption secondary to intravenous acyclovir. Acta Derm Venereol. 2008;88(2):196-198.

25. Soul-Lawton J, Seaber E, On N, Wootton R, Rolan P, Posner J. Absolute bioavailability and metabolic disposition of valaciclovir, the L-valyl ester of acyclovir, following oral administration to humans. Antimicrob Agents Chemother. 1995;39(12):2759-2764.

26. De Clercq E, Field HJ. Antiviral prodrugs - the development of successful prodrug strategies for antiviral chemotherapy. Br J Pharmacol. 2006;147(1):1-11.

27. Bayrou O, Gaouar H, Leynadier F. Famciclovir as a possible alternative treatment in some cases of allergy to acyclovir. Contact Dermatitis. 2000;42(1):42.

28. Lea AP, Bryson HM. Cidofovir. Drugs. 1996;52(2):225230; discussion 231.

29. Razonable RR. Antiviral drugs for viruses other than human immunodeficiency virus. Mayo Clin Proc. 2011;86(10):1009-1026.

30. Kim SJ, Kim K, Do YR, Bae SH, Yang DH, Lee JJ. Low-dose acyclovir is effective for prevention of herpes zoster in myeloma patients treated with bortezomib: a report from the Korean Multiple Myeloma Working Party (KMMWP) Retrospective Study. Jpn J Clin Oncol. 2011;41(3):353-357. 